\title{
Paramedic Recognition of Sepsis in the Prehospital Setting: A Prospective Observational Study
}

\author{
Robert S. Green, ${ }^{1,2}$ Andrew H. Travers, ${ }^{3,4}$ Edward Cain, ${ }^{3}$ Samuel G. Campbell, \\ Jan L. Jensen, ${ }^{3,4}$ David A. Petrie, ${ }^{3}$ Mete Erdogan, ${ }^{2}$ Gredi Patrick, ${ }^{5}$ and Ward Patrick ${ }^{1}$ \\ ${ }^{1}$ Department of Critical Care, Dalhousie University, Suite 377, Bethune Building, 1276 South Park Street, Halifax, \\ NS, Canada B3H 2 Y9 \\ ${ }^{2}$ Trauma Nova Scotia, Room 1-026B, Centennial Building, 1276 South Park Street, Halifax, NS, Canada B3H 2 Y9 \\ ${ }^{3}$ Department of Emergency Medicine, Dalhousie University, Division of EMS, 1796 Summer Street, Halifax Infirmary, \\ Suite 355, Halifax, NS, Canada B3H 3A7 \\ ${ }^{4}$ Emergency Health Service, 239 Brownlow Avenue, Suite 300, Dartmouth, NS, Canada B3B 2B2 \\ ${ }^{5}$ Performance Excellence, Nova Scotia Health Authority, Halifax, NS, Canada B3H 4R2 \\ Correspondence should be addressed to Robert S. Green; greenrs@dal.ca
}

Received 22 December 2015; Accepted 17 February 2016

Academic Editor: Rade B. Vukmir

Copyright (c) 2016 Robert S. Green et al. This is an open access article distributed under the Creative Commons Attribution License, which permits unrestricted use, distribution, and reproduction in any medium, provided the original work is properly cited.

\begin{abstract}
Background. Patients with sepsis benefit from early diagnosis and treatment. Accurate paramedic recognition of sepsis is important to initiate care promptly for patients who arrive by Emergency Medical Services. Methods. Prospective observational study of adult patients (age $\geq 16$ years) transported by paramedics to the emergency department (ED) of a Canadian tertiary hospital. Paramedic identification of sepsis was assessed using a novel prehospital sepsis screening tool developed by the study team and compared to blind, independent documentation of ED diagnoses by attending emergency physicians (EPs). Specificity, sensitivity, accuracy, positive and negative predictive value, and likelihood ratios were calculated with $95 \%$ confidence intervals. Results. Overall, 629 patients were included in the analysis. Sepsis was identified by paramedics in $170(27.0 \%)$ patients and by EPs in $71(11.3 \%)$ patients. Sensitivity of paramedic sepsis identification compared to EP diagnosis was $73.2 \%$ (95\% CI 61.4-83.0), while specificity was $78.8 \%$ (95\% CI 75.2-82.2). The accuracy of paramedic identification of sepsis was $78.2 \%(492 / 629,52$ true positive, 440 true negative). Positive and negative predictive values were 30.6\% (95\% CI 23.8-38.1) and 95.9\% (95\% CI 93.6-97.5), respectively. Conclusion. Using a novel prehospital sepsis screening tool, paramedic recognition of sepsis had greater specificity than sensitivity with reasonable accuracy.
\end{abstract}

\section{Introduction}

Despite numerous clinical trials of specific treatments, sepsis remains a leading cause of morbidity and mortality in patients arriving at the emergency department (ED) or staying in hospital $[1,2]$. Early detection and protocol-based care (PBC) of sepsis with resuscitation, antibiotics, and source control are considered the optimal management strategy [3-6]. Of patients seen in the ED with sepsis, approximately half are transported there by Emergency Medical Services (EMS) [7]. Previous studies have demonstrated that septic patients who arrive by EMS receive treatment rapidly compared to ambulatory patients $[8,9]$, as with other time sensitive conditions such as myocardial infarction $[10,11]$.
Paramedic recognition of sepsis is important for the appropriate triage and initiation of early treatment for septic patients transported by EMS. However, identification of sepsis can be challenging for paramedics since they cannot access the laboratory and imaging adjuncts available to physicians in the ED. There is limited information available on how well paramedics are able to identify sepsis compared to emergency physicians (EPs) [12-14], and few screening tools have been developed to assist with prehospital recognition of sepsis [1417].

Most previous screening tools have focused on detection of patients with severe sepsis, and only one of these tools has been validated to date [15]. Importantly, these screening tools incorporate limited information regarding recent findings of 
infection in patients and criteria of organ dysfunction remote from the site of infection. To address these limitations, the study team developed a novel prehospital tool to screen for patients with any degree of sepsis. The goal of this study was to evaluate use of this tool by EMS personnel and to compare paramedic detection of septic patients with EP diagnoses.

\section{Methods}

This prospective observational study was of a convenience sample of adult patients (age $\geq 16$ years) transported by paramedics to a Canadian tertiary ED. Patients were enrolled into the study by paramedics if they were dispatched as follows: having abdominal pain, having breathing problems, being sick, having unknown problems, being unconscious/fainting, having chest pain, or any case in which paramedics considered sepsis a possible diagnosis. A novel paper-based prehospital sepsis screening tool was developed by the study team. Relevant data points were determined through literature searches related to diagnosis of sepsis, in addition to consensus or guideline statements. The prehospital sepsis screening tool is available in Supplementary Material available online at http://dx.doi.org/10.1155/2016/6717261.

The content of the tool relied heavily upon sepsis criteria definitions developed by the 2001 SCCM/ESICM/ACCP/ ATS/SIS sepsis definitions conference [18]. Possible data elements were vetted through use of a Delphi approach with provincial leaders in paramedicine, emergency medicine, and critical care medicine. The tool was evaluated by a focus group of 40 EMS paramedics to obtain feedback and ensure that data elements could be obtained in the prehospital environment without delaying the implementation of the current standard of care to patients. Prior to study initiation, paramedics attended a 3-hour training session and received instruction on sepsis including definitions, epidemiology, basic pathophysiology, identification, and optimal management of septic patients.

In this Canadian provincial ground ambulance system, most paramedics are primary care paramedics (PCPs) or advanced care paramedics (ACPs) (http://novascotia.ca/dhw/ ehs/international-applicants/levels-of-paramedics.asp). PCP training is one to two years in length. PCPs can provide basic level emergency care, including performing semiautomated external defibrillation, oxygen administration, intravenous therapy, cardiac monitoring, and administration of select medications. ACP training is 18 to 36 months in length. ACPs are able to provide advanced level emergency care, including a broad array of emergency medications, advanced airway management, and invasive interventions. Training requirements follow the National Occupational Competency Profile (http://www.paramedic.ca/site/nocp?nav=02).

Immediately following transport to hospital and transfer of care to the ED, paramedics were prompted by the EMS dispatch center to complete the prehospital sepsis screening tool. The tool was used to collect data on patient history, vital signs, recent findings (within past 10 days), signs and symptoms, criteria for organ dysfunction remote from the site of infection, and presence of fever. The determination of
TABLE 1: Paramedic certification level.

\begin{tabular}{lc}
\hline & Frequency \\
\hline Advanced care paramedic & $257(43.8)$ \\
Primary care paramedic & $219(37.4)$ \\
Intermediate care paramedic & $97(16.6)$ \\
Critical care paramedic & $13(2.2)$ \\
\hline
\end{tabular}

Parentheses denote percentage.

$n=586$ responses.

whether a patient had a fever was based on self-reporting by the patient or the presence of symptoms such as abnormally warm forehead/neck, flushed face, lethargy, and body aches; thermometers were not routinely used by EMS personnel at the time of this study. Based on data collected using the tool, paramedics made a determination of whether or not the patient had any degree of sepsis which was compared to blind, independent documentation of diagnoses by attending EPs.

The EPs used a study ED screening tool to indicate if patients were septic, which served as the gold standard for this study. If the EP did not mark whether or not the patient had sepsis, the final EP diagnosis recorded on the patients' medical record was obtained through retrospective review of the electronic ED database and study investigators determined if the final diagnosis indicated that the patient had sepsis. Data was entered into a dedicated study database (Microsoft ${ }^{\circledR}$ Access, Redmond WA). Analysis was conducted in IBM SPSS Statistics Version 21 (IBM, Armonk NY) [19]. Simple descriptive statistics were used to describe characteristics of paramedics and the patient cohort. Specificity, sensitivity, accuracy, positive and negative predictive value, and likelihood ratios were calculated with 95\% confidence intervals. This study was approved by the Institutional Review Board.

\section{Results}

Overall, 956 patients were enrolled by paramedics during the study period. A flow diagram outlining the selection of study participants and recognition of sepsis by paramedics and EPs is shown in Figure 1. We excluded 327 cases with no paramedic diagnosis recorded $(n=249)$, no EP diagnosis available ( $n=73$ ), or if the patient left the ED without being seen or before the EP diagnosis was made $(n=5)$. The remaining 629 (65.8\%) cases were included in the final analysis.

The certification level of paramedics who participated in the study is shown in Table 1 . In most cases, the EMS personnel were an ACP (43.8\%) or a PCP (37.4\%). Characteristics of the patients seen by paramedics are described in Table 2. Patients commonly presented with fever $(27.9 \%)$ and with a recent infection within the prior 10 days (25.2\%). Paramedics identified 170/629 (27.0\%) patients as septic, while EPs identified sepsis in 71/629 (11.3\%) patients. Disagreement in the diagnosis of sepsis often occurred in patients ultimately diagnosed with pneumonia, cellulitis, urinary tract infection, peritonitis, and renal failure. 


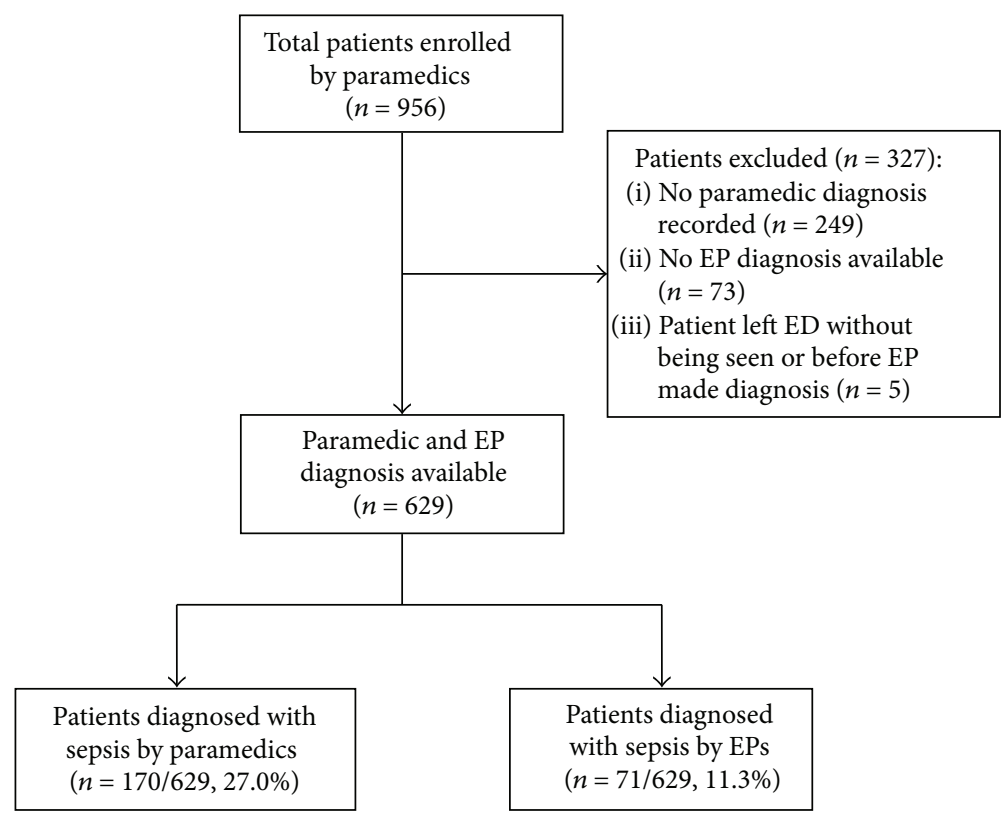

FIGURE 1: Selection of study participants and identification of sepsis by paramedics and EPs. ED, emergency department; EP, emergency physician.

Table 3 shows paramedic identification of sepsis and EP diagnosis of sepsis as a contingency table. The sensitivity and specificity of paramedic diagnoses of sepsis compared to EP diagnoses were $73.2 \%$ (95\% CI 61.4-83.0) and 78.8\% (95\% CI 75.2-82.2), respectively. Accuracy was 78.2\% (492/629, 52 true positive, 440 true negative). The positive predictive value was $30.6 \%$ (95\% CI 23.8-38.1), and the negative predictive value was $95.9 \%$ (95\% CI 93.6-97.5). The positive likelihood ratio was 3.46 (95\% CI 2.80-4.29), and negative likelihood ratio was 0.34 (95\% CI: $0.23-0.50$ ).

\section{Discussion}

This prospective study assessed the ability of paramedics to recognize septic patients using a novel prehospital sepsis screening tool. Compared with the EPs' diagnosis, we observed that paramedics were able to identify septic patients with $73.2 \%$ sensitivity, $78.8 \%$ specificity, and $78.2 \%$ accuracy using this tool. This study provides evidence that paramedics can identify sepsis with reasonable accuracy in the EMS setting. Increasing paramedic awareness of the importance of early recognition through directed training is critical to reducing the burden of sepsis-related morbidity and mortality.

Few previous studies have examined the ability of prehospital personnel to recognize sepsis in adult patients. Using presence of both an ED report of acute infection and patient admission as their reference standard, one prospective study employed a questionnaire to examine the ability of EMS providers to identify serious infection and reported a sensitivity of $31 \%$ (95\% CI 17-50) and specificity of $93 \%$ (95\% CI 87-96) [20]. Other studies have found the sensitivity of sepsis identification by prehospital personnel to be $70 \%$ or higher [21, 22], similar to the results of our study.

There are limited reports of validated screening tools for recognition of sepsis in the prehospital setting. One tool recently developed and validated is the prehospital severe sepsis (PRESS) screening tool which involves a score calculated from EMS data on 6 risk factors [15]. Using the PRESS tool in the setting of an urban public hospital, prehospital recognition of severe sepsis by EMS staff had a sensitivity of $86 \%$ and a specificity of $47 \%$ [15]. The Sepsis Alert Protocol is another screening tool for EMS providers and was found to accurately identify severe sepsis in $47.8 \%$ of cases in a pilot study [14]. Unlike these tools, our prehospital screening tool was designed to identify patients with any degree of sepsis, not only those with severe sepsis. Comparing the accuracy of our tool with these other tools is difficult due to variations in study designs, institutional settings, EMS services, and populations served. Additional validation is required before these tools can be recommended for widespread clinical use.

Our study evaluated the ability of paramedics to identify sepsis using a prehospital screening tool and found promising levels of accuracy, sensitivity, and specificity ( $>70 \%)$. These findings are significant in order to capitalize on early alerts to further reduce the time window to treatment initiation [23]. Additional areas for future research may involve integrating the use of biomarkers and evaluating the impact of moving ED treatments earlier into the EMS phase of care. Another opportunity that is yet unexplored is the identification of patients who may have sepsis by EMS dispatchers [24]. There is tremendous potential to reduce the morbidity and mortality associated with sepsis by developing and validating new tools for early recognition and management of the septic patient. 
TABLE 2: Patient characteristics.

\begin{tabular}{|c|c|}
\hline & Frequency \\
\hline \multicolumn{2}{|l|}{ Patient history $^{1}$} \\
\hline Diabetes mellitus & $117(21.1)$ \\
\hline Oral steroids or chemotherapy in last 6 weeks & $38(6.8)$ \\
\hline Chronic renal failure & $23(4.1)$ \\
\hline Organ/tissue transplant & $5(0.9)$ \\
\hline Human immunodeficiency virus & $1(0.2)$ \\
\hline None of the above & $394(71.0)$ \\
\hline \multicolumn{2}{|l|}{ Vital signs, mean [SD] } \\
\hline Respiratory rate & $21.6[8.3]$ \\
\hline Heart rate & 90.9 [22.6] \\
\hline Systolic blood pressure ${ }^{2}$ & $132.3[29.5]$ \\
\hline Diastolic blood pressure $^{3}$ & $72.8[14.8]$ \\
\hline Blood sugar $^{4}$ & $8.1[3.3]$ \\
\hline \multicolumn{2}{|l|}{ Fever at present ${ }^{5}$} \\
\hline Yes & $163(27.9)$ \\
\hline \multicolumn{2}{|l|}{ Recent finding of any infection ${ }^{6}$} \\
\hline Yes & $155(25.2)$ \\
\hline \multicolumn{2}{|l|}{ Signs and symptoms of infection ${ }^{7}$} \\
\hline Pulmonary, cough/hypoxia & $170(27.5)$ \\
\hline Abdominal tenderness & $81(13.1)$ \\
\hline Soft tissue swelling/redness/pain & $62(10.0)$ \\
\hline Altered sensorium & $56(9.0)$ \\
\hline Urinary, foul/puss & $52(8.4)$ \\
\hline Meningitis, stiff neck & $5(0.8)$ \\
\hline None of the above & $259(41.8)$ \\
\hline \multicolumn{2}{|l|}{ Organ dysfunction criteria ${ }^{8}$} \\
\hline Diaphoresis & $47(7.8)$ \\
\hline Cool peripheral limbs & $38(6.3)$ \\
\hline Systolic blood pressure $<90 \mathrm{mmHg}$ & $36(6.0)$ \\
\hline Knee mottling & $1(0.2)$ \\
\hline Periumbilical mottling & $0(0)$ \\
\hline None of the above & $498(82.7)$ \\
\hline
\end{tabular}

SD: standard deviation

Brackets denote standard deviation. Parentheses denote percentage.

${ }^{1}$ Paramedics could make more than one selection for patient history, $n=555$ responses.

${ }^{2} n=627$ responses.

${ }^{3}$ Diastolic blood pressure unavailable when palpation was required, $n=554$ responses.

${ }^{4}$ Blood sugar was often unavailable and only collected in nondiabetic patients, $n=446$ responses.

${ }^{5}$ Paramedic determination of whether patient had a fever at present, $n=584$ responses.

${ }^{6}$ Paramedic finding of any infection within prior 10 days, $n=614$ responses.

${ }^{7}$ Any sign or symptom of infection (both present and new). Paramedics could make more than one selection, $n=619$ responses.

${ }^{8}$ Any criteria of organ dysfunction present and remote from site of infection that were not chronic conditions. Paramedics could make more than one selection, $n=602$ responses.

This study has the limitations of an observational study including possible selection and information bias. This study was conducted at a single academic tertiary care center $(65,000 \mathrm{ED}$ visits per year) with a single EMS service that takes care of urban, suburban, and rural populations; thus,
TABLE 3: Contingency table comparing paramedic identification of sepsis with emergency physician diagnosis of sepsis.

\begin{tabular}{|c|c|c|}
\hline & \multicolumn{2}{|c|}{$\begin{array}{l}\text { Emergency physician } \\
\text { diagnosis of sepsis }\end{array}$} \\
\hline & Yes & No \\
\hline \multicolumn{3}{|c|}{ Paramedic identification of sepsis } \\
\hline Yes & 52 & 118 \\
\hline No & 19 & 440 \\
\hline
\end{tabular}

our findings may not be generalizable to other centers. While we did capture paramedic certification level, data on number of years of experience for paramedics and EPs was not collected. While this study is limited because the reference standard was EP diagnosis instead of a more definitive marker, this reference was chosen as EP diagnosis is the sentinel trigger of $\mathrm{PBC}$ at our institution. Another limitation of this study is the number of potentially eligible patients that were excluded, mostly because paramedics did not complete the diagnosis on the study form. At the time of the study, this EMS system did not use thermometers or point of care lactate testing, which may limit generalizability to settings that do use these diagnostic tools.

\section{Conclusion}

Using a novel screening tool, paramedic identification of sepsis in this limited single center study cohort revealed a greater specificity than sensitivity in comparison to EP diagnosis. Further research is required to validate the use of this sepsis screening tool in the prehospital setting.

\section{Conflict of Interests}

The authors declare that there is no conflict of interests regarding the publication of this paper.

\section{Acknowledgments}

The authors would like to thank John Kasemets (CCP) for his significant contribution to study design, David Urqhart for developing the study database, and Emily Morash, Maureen Sturgeon (ACP), and Zach Dewar (PCP) for their work on data entry and chart reviews.

\section{References}

[1] D. F. Gaieski, J. M. Edwards, M. J. Kallan, and B. G. Carr, "Benchmarking the incidence and mortality of severe sepsis in the united states," Critical Care Medicine, vol. 41, no. 5, pp. 11671174, 2013.

[2] J. C. Marshall, "Why have clinical trials in sepsis failed?" Trends in Molecular Medicine, vol. 20, no. 4, pp. 195-203, 2014.

[3] R. P. Dellinger, M. N. Levy, A. Rhodes et al., "Surviving sepsis campaign: international guidelines for management of severe sepsis and septic shock: 2012," Critical Care Medicine, vol. 41, no. 2, pp. 580-637, 2013. 
[4] The ProCESS Investigators, "A randomized trial of protocolbased care for early septic shock," The New England Journal of Medicine, vol. 370, no. 18, pp. 1683-1693, 2014.

[5] D. M. Yealy, D. T. Huang, A. Delaney et al., "Recognizing and managing sepsis: what needs to be done?" BioMed Central Medicine, vol. 27, no. 13, article 98, 2015.

[6] R. L. Gauer, "Early recognition and management of sepsis in adults: the first six hours," American Family Physician, vol. 88, no. 1, pp. 44-53, 2013.

[7] C. W. Seymour, T. D. Rea, J. M. Kahn, A. J. Walkey, D. M. Yealy, and D. C. Angus, "Severe sepsis in pre-hospital emergency care: analysis of incidence, care, and outcome," American Journal of Respiratory and Critical Care Medicine, vol. 186, no. 12, pp. 12641271, 2012.

[8] R. A. Band, D. F. Gaieski, J. H. Hylton, F. S. Shofer, M. Goyal, and Z. F. Meisel, "Arriving by emergency medical services improves time to treatment endpoints for patients with severe sepsis or septic shock," Academic Emergency Medicine, vol. 18, no. 9, pp. 934-940, 2011.

[9] J. R. Studnek, M. R. Artho, C. L. Garner Jr., and A. E. Jones, “The impact of emergency medical services on the ED care of severe sepsis," The American Journal of Emergency Medicine, vol. 30, no. 1, pp. 51-56, 2012.

[10] T. Camp-Rogers, S. Dante, M. C. Kontos, C. S. Roberts, L. Kreisa, and M. C. Kurz, "The impact of prehospital activation of the cardiac catheterization team on time to treatment for patients presenting with ST-segment-elevation myocardial infarction," The American Journal of Emergency Medicine, vol. 29, no. 9, pp. 1117-1124, 2011.

[11] R. A. Ducas, A. W. Wassef, D. S. Jassal et al., “To transmit or not to transmit: how good are emergency medical personnel in detecting STEMI in patients with chest pain?" The Canadian Journal of Cardiology, vol. 28, no. 4, pp. 432-437, 2012.

[12] M. Groenewoudt, A. A. Roest, F. M. M. Leijten, and P. M. Stassen, "Septic patients arriving with emergency medical services: a seriously ill population," The European Journal of Emergency Medicine, vol. 21, no. 5, pp. 330-335, 2014.

[13] C. W. Seymour, C. R. Cooke, S. R. Heckbert et al., "Prehospital intravenous access and fluid resuscitation in severe sepsis: an observational cohort study," Critical Care, vol. 18, no. 5, article 533, 2014.

[14] W. F. Guerra, T. R. Mayfield, M. S. Meyers, A. E. Clouatre, and J. C. Riccio, "Early detection and treatment of patients with severe sepsis by prehospital personnel," The Journal of Emergency Medicine, vol. 44, no. 6, pp. 1116-1125, 2013.

[15] C. C. Polito, A. Isakov, A. H. Yancey II et al., "Prehospital recognition of severe sepsis: development and validation of a novel EMS screening tool," The American Journal of Emergency Medicine, vol. 33, no. 9, pp. 1119-1125, 2015.

[16] U. M. Wallgren, M. Castrén, A. E. V. Svensson, and L. Kurland, "Identification of adult septic patients in the prehospital setting: a comparison of two screening tools and clinical judgment," The European Journal of Emergency Medicine, vol. 21, no. 4, pp. 260265, 2014.

[17] O. Bayer, D. Schwarzkopf, C. Stumme et al., "An early warning scoring system to identify septic patients in the prehospital setting: the PRESEP score," Academic Emergency Medicine, vol. 22, no. 7, pp. 868-871, 2015.

[18] M. M. Levy, M. P. Fink, J. C. Marshall et al., "2001 SCCM/ ESICM/ACCP/ATS/SIS international sepsis definitions conference," Critical Care Medicine, vol. 31, no. 4, pp. 1250-1256, 2003.
[19] IBM Corp, IBM SPSS Statistics for Windows, Version 21.0, IBM Corp, Armonk, NY, USA, 2012.

[20] B. Suffoletto, A. Frisch, A. Prabhu, J. Kristan, F. X. Guyette, and C. W. Callaway, "Prediction of serious infection during prehospital emergency care," Prehospital Emergency Care, vol. 15, no. 3, pp. 325-330, 2011.

[21] C. W. Seymour, C. R. Cooke, M. E. Mikkelsen et al., "Out-ofhospital fluid in severe sepsis: effect on early resuscitation in the emergency department," Prehospital Emergency Care, vol. 14, no. 2, pp. 145-152, 2010.

[22] K. Clesham, S. Mason, J. Gray, S. Walters, and V. Cooke, "Can emergency medical service staff predict the disposition of patients they are transporting?" Emergency Medicine Journal, vol. 25, no. 10, pp. 691-694, 2008.

[23] A. A. Báez, P. Hanudel, M. T. Perez, E. M. Giraldez, and S. R. Wilcox, "Prehospital sepsis project (PSP): knowledge and attitudes of united states advanced out-of-hospital care providers," Prehospital and Disaster Medicine, vol. 28, no. 2, pp. 104-106, 2013.

[24] J. Herlitz, A. Bång, B. Wireklint-Sundström et al., "Suspicion and treatment of severe sepsis. An overview of the prehospital chain of care," Scandinavian Journal of Trauma, Resuscitation and Emergency Medicine, vol. 20, article 42, 2012. 


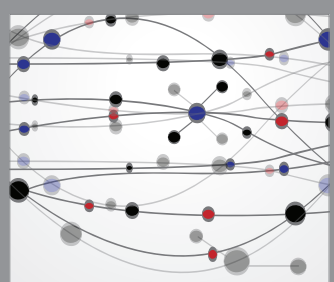

The Scientific World Journal
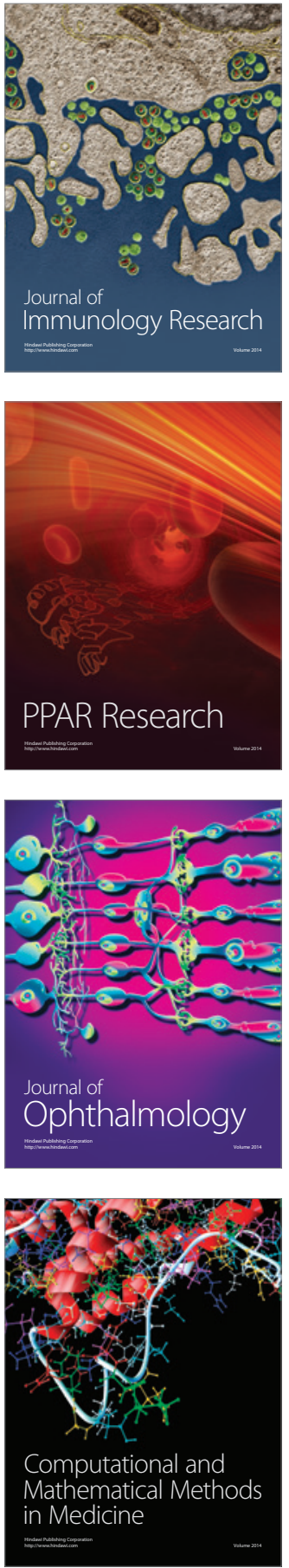

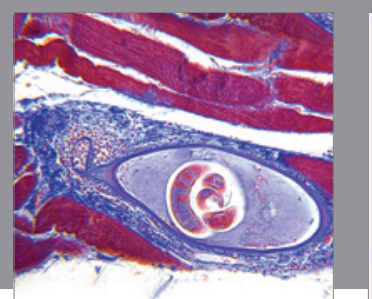

Gastroenterology Research and Practice

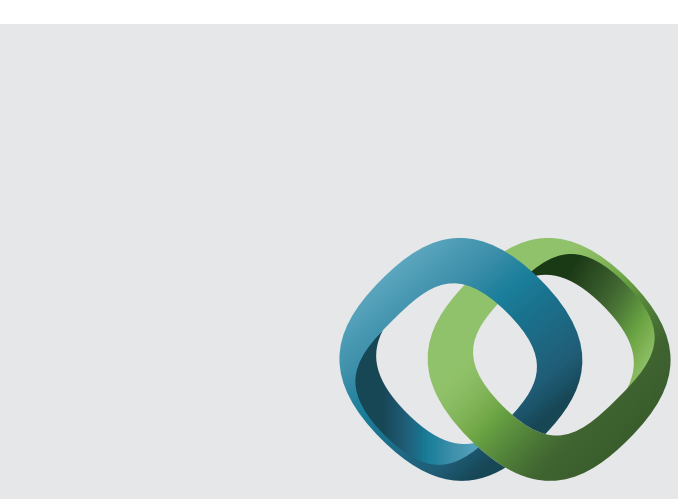

\section{Hindawi}

Submit your manuscripts at

http://www.hindawi.com
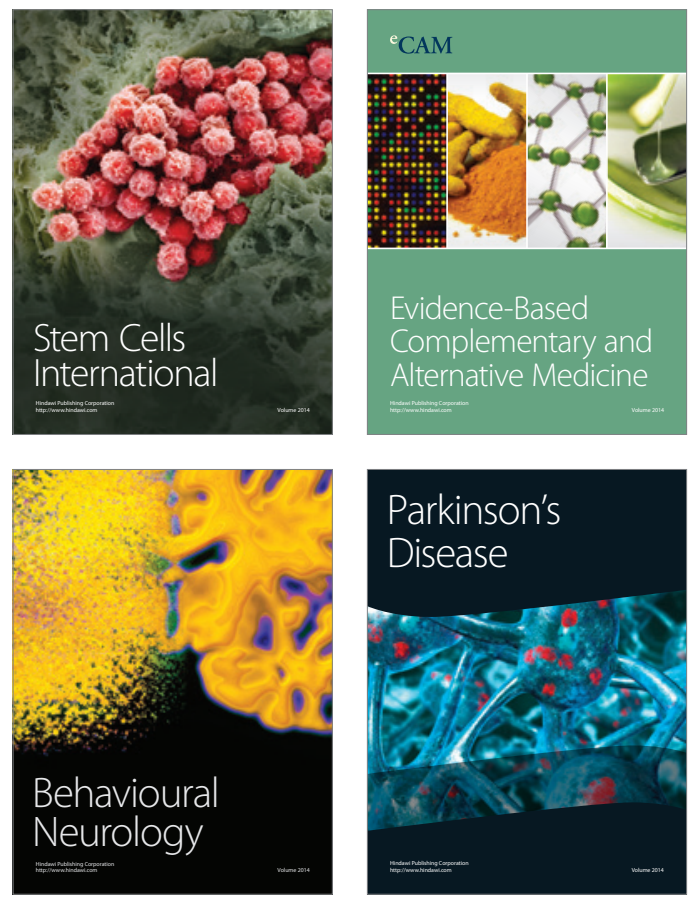
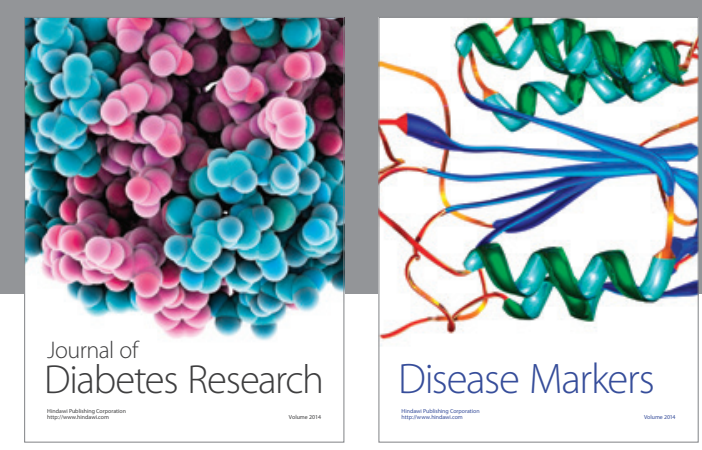

Disease Markers
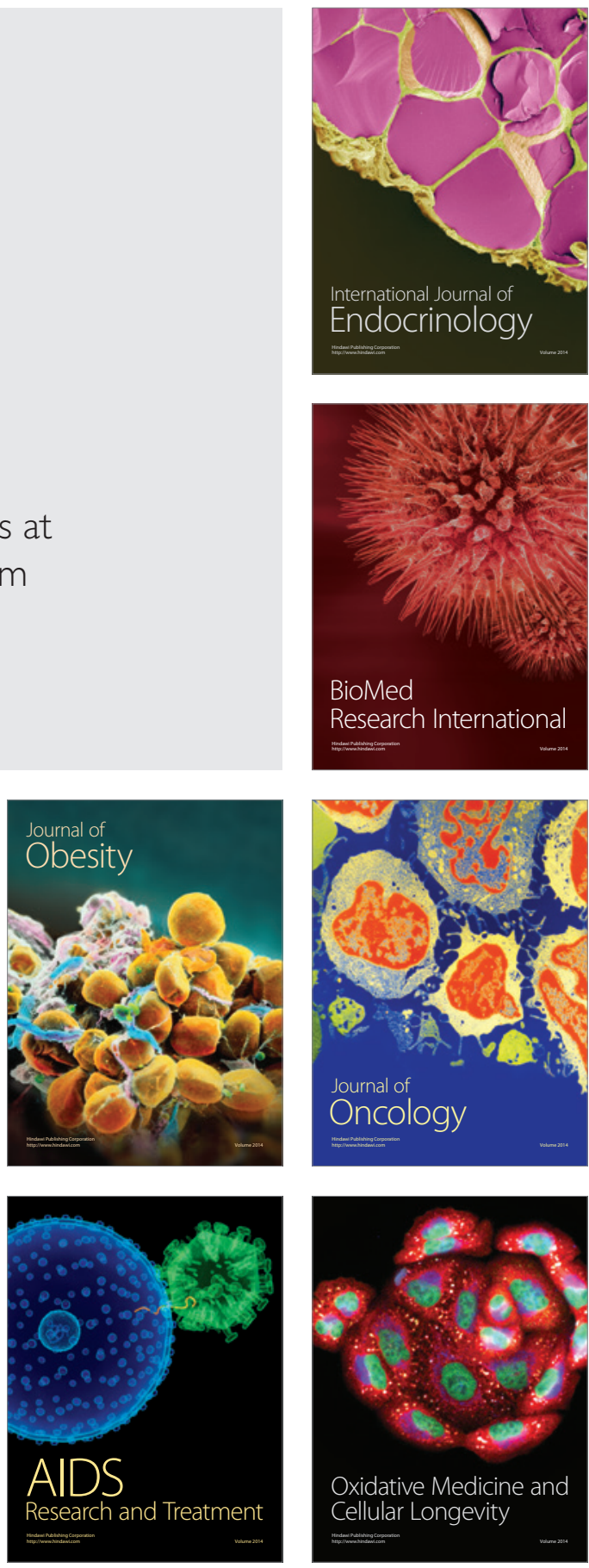